\title{
HEMODYNAMIC UNLOADING LEADS TO REGRESSION OF PULMONARY VASCULAR DISEASE IN RATS
}

Stacy B. O'Blenes, MD, MSc ${ }^{\mathrm{a}}$

Stefan Fischer, MD ${ }^{\mathrm{b}}$

Brendan McIntyre, BSc ${ }^{\mathrm{a}}$

Shaf Keshavjee, MD, MSc ${ }^{\mathrm{b}}$

Marlene Rabinovitch, $\mathrm{MD}^{\mathrm{a}}$
From The Division of Cardiovascular Research, Departments of Pediatrics, Laboratory Medicine and Pathobiology, and Medicine, The Hospital for Sick Children, University of Toronto, ${ }^{\mathrm{a}}$ and the Thoracic Surgery Research Laboratory, The Toronto General Hospital Research Institute, ${ }^{\mathrm{b}}$ Toronto, Ontario, Canada.

Supported by grants from the Heart and Stroke Foundation of Canada, the Medical Research Council of Canada, and the Primary Pulmonary Hypertension Cure Foundation.

Read at the Eightieth Annual Meeting of The American Association for Thoracic Surgery, Toronto, Ontario, Canada, April 30-May 3, 2000 .
Objective: Treatment options for patients with advanced pulmonary vascular disease caused by a congenital heart defect are still mainly limited to hearttransplantation or lung transplantation with repair of the cardiac lesion we have previously shown that the structural changes associated pulmonary hypertension can be reversed by stress unloading in an organ Methods: Right middle and lower lobectomy and monocrotaline injection were performed in Lewis rats $(n=22)$ to cause pulmonary vascular disease from a combined hemodynamic and toxic injury. Twenty-eight days later the left lungs were examined $(n=10)$ or exposed to normal pulmonary artery pressure for an additional $14(\mathrm{n}=5)$ or $28(\mathrm{n}=7)$ days by transplantation into healthy recipients. Pulmonary artery pressure, ventricular weight, and pulmonary artery morphology were evaluated in each group.

Results: Pulmonary hypertension (50 vs $16 \mathrm{~mm} \mathrm{Hg} ; P<.001$ ) and right ventricular hypertrophy (right ventricular/left ventricular weight 0.69 vs 0.32 ; $P<.001)$ associated with pulmonary artery medial hypertrophy $(28.2 \% \mathrm{vs}$ $7.2 \%$ wall thickness; $P<.001)$ and muscularization of small pulmonary arteries $(92.3 \%$ vs $19.4 \% ; P<.001)$ developed by day 28 (compared with untreated controls). However, transplantation into healthy recipients effectively unloaded the lungs (mean pulmonary artery pressure 17 and $24 \mathrm{~mm}$ $\mathrm{Hg}$ at 14 and 28 days after transplantation) and resulted in progressive normalization of medial hypertrophy (15.6\% and $12.1 \%$ at 14 and 28 days) and muscularization $(65.1 \%$ and $42.2 \%$ at 14 and 28 days) relative to nontransplanted controls $(P<.005$ in each case $)$.

Conclusions: Hemodynamic unloading of lungs with pulmonary vascular disease results in progressive normalization of pulmonary artery structure. These results are the first to provide a rationale for attempting to induce regression of pulmonary vascular disease by pressure unloading of the pulmonary circulation. Methods to mechanically unload the pulmonary circulation should be critically evaluated as a strategy for staged surgical repair of congenital heart defects despite presumed irreversible pulmonary hypertension. (J Thorac Cardiovasc Surg 2001;121:279-89)
Received for publication May 4, 2000; revisions requested July 18, 2000; revisions received Aug 4, 2000; accepted for publication Sept 14, 2000.

Address for reprints: Marlene Rabinovitch, MD, Division of Cardiovascular Research, The Hospital for Sick Children, 555 University Ave, Toronto, Ontario, Canada, M5G 1X8 (E-mail: mr@sickkids.on.ca).

Copyright (ㄷ 2001 by The American Association for Thoracic Surgery

$0022-5223 / 2001 \$ 35.00+0 \quad \mathbf{1 2 / 6 / 1 1 1 6 5 7}$

doi:10.1067/mtc.2001.111657 
0 bstructive pulmonary vascular disease caused by a chronic left-to-right shunt complicates certain congenital heart defects if not repaired early in life. Elevated and fixed pulmonary vascular resistance predisposes the patient to postoperative pulmonary hypertension and right ventricular (RV) failure and may make the risk of surgical correction prohibitive. The treatment options for patients with advanced pulmonary vascular disease associated with a congenital heart defect are currently limited to heart-lung transplantation or lung transplantation and simultaneous intracardiac repair. ${ }^{1-4}$ Long-term intravenous epoprostenol (formerly, prostacyclin) infusion may reduce pulmonary vascular resistance and improve the hemodynamics in this group of patients. ${ }^{5}$ However, a method of reversing the structural changes in the pulmonary circulation may allow surgical repair of the congenital heart defect with an acceptable risk.

Obstructive pulmonary vascular disease in patients with a congenital heart defect is characterized by thickening of the medial layer of larger muscular arteries as a result of smooth muscle cell proliferation and hypertrophy. Furthermore, the medial smooth muscle layer extends to the smaller, normally nonmuscular peripheral pulmonary arteries (PAs). These features, along with neointimal proliferation and obliteration of peripheral PAs associated with reduced arterial density, all contribute to the increase in pulmonary vascular resistance. ${ }^{6-9}$ We have previously demonstrated, in an organ culture model, that unloading mechanical stress reverses the structural changes associated with pulmonary hypertension by triggering smooth muscle cell apoptosis and extracellular matrix resorption..$^{10}$ Improvement in the biopsy findings in patients with pulmonary vascular disease after banding of the PA has been reported in a few patients ${ }^{11}$ and could similarly be due to an unloading of hemodynamic forces.

To test the hypothesis that hemodynamic unloading leads to regression of the structural changes associated with obstructive pulmonary vascular disease, we adapted an animal model of pulmonary hypertension. Increased pulmonary blood flow induced by pulmonary resection and endothelial injury caused by the toxin monocrotaline in the rat result in PA changes that share many features with the human disease..$^{12,13}$ To model hemodynamic unloading in vivo, we transplanted lungs from rats with pulmonary hypertension into normotensive recipient rats and examined them 14 and 28 days later to evaluate regression of the structural changes. Our data indicate that hemodynamic unloading of lungs with pulmonary vascular disease leads to a progressive normalization of the structural changes associated with pulmonary vascular disease including medial hypertrophy and muscularization of peripheral PAs. Regression of the pulmonary vascular changes is associated with a reduction in cellular proliferation rates. These data support the hypothesis that obstructive pulmonary vascular disease can be reversed by hemodynamic unloading. If a method of safely unloading the pulmonary circulation can be devised, it may allow for staged repair of congenital heart defects with acceptable risk despite presumed irreversible pulmonary vascular disease.

\section{Methods}

Experimental design. Forty-one syngeneic adult Lewis rats (Charles River Laboratories, St Laurent, Quebec, Canada) with an average body weight of $358 \pm 8 \mathrm{~g}$ were randomly selected as untreated controls $(n=4)$ or subjected to right middle and lower lobectomy followed by injection of monocrotaline $(n=37)$ to induce pulmonary hypertension. Twenty-eight days after right lung resection and monocrotaline injection, left lungs from the surviving rats $(\mathrm{n}=30)$ were harvested for morphometric evaluation $(n=10)$ or exposed to normal PA pressures by orthotopic transplantation into healthy normotensive recipients $(n=20)$. Because monocrotaline-induced pulmonary vascular disease is progressive and fatal beyond 28 days, ${ }^{14}$ the remaining lungs $(\mathrm{n}=$ 10) were also harvested on day 28 and served as a control group. The transplanted left lungs were then recovered from the surviving recipients $14(\mathrm{n}=5)$ or $28(\mathrm{n}=7)$ days after transplantation. PA pressure, RV hypertrophy, PA morphology, and cellular proliferation were evaluated in each group. All animals received standard postoperative care according to protocols approved by the Animal Care Committee of the Hospital for Sick Children and in compliance with guidelines from the Canadian National Society for Medical Research.

Anesthesia and postoperative care. Rats were sedated by an intraperitoneal injection of ketamine $(7 \mathrm{mg} / \mathrm{kg}$; ParkeDavis, Toronto, Ontario, Canada), anesthetized with isoflurane (Zeneca, Mississauga, Ontario, Canada) and nitrous oxide, and then intubated endotracheally with a 14-gauge intravenous catheter. The animals' lungs were ventilated with a volume-controlled ventilator (Harvard rodent ventilator, model 683; Harvard Apparatus Company, S Natick, Mass) at a rate of 80 breaths $/ \mathrm{min}$ and a tidal volume of $1 \mathrm{~mL} / 100 \mathrm{~g}$ with a positive end-expiratory pressure of $2 \mathrm{~cm} \mathrm{H}_{2} \mathrm{O}$. The electrocardiogram was continuously monitored. An 18-gauge intravenous catheter attached to an underwater seal and suction was used to evacuate the pleural space on closure of the thoracotomy incision. At the termination of the surgical procedure, the animals were ventilated with $100 \%$ oxygen and allowed to awaken. When spontaneous breathing was observed, the animals were extubated and the pleural catheter removed. Buprenorphine $(0.05 \mathrm{mg} / \mathrm{kg}$; Reckitt \& Coleman Pharmaceutical Inc, Richmond, Va) was administered for pain control by intraperitoneal injection immediately after extubation and as required during the postoperative period. 

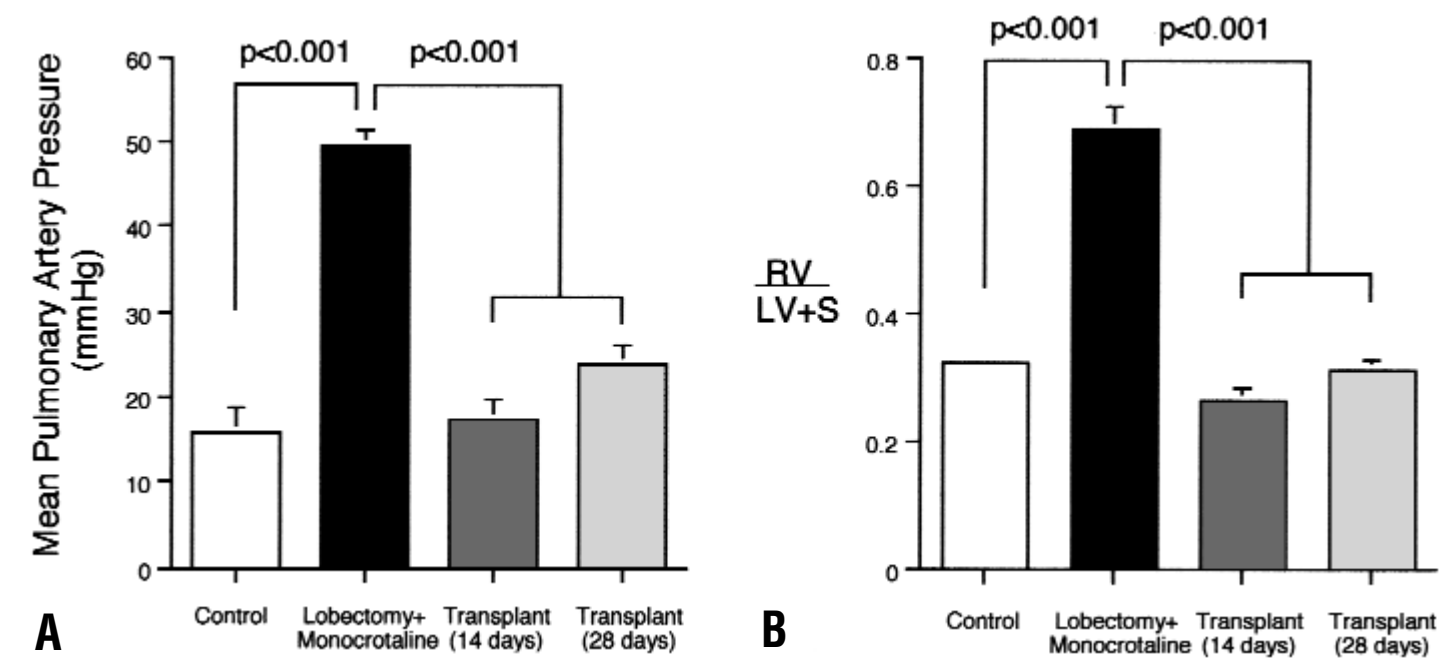

Fig 1. PA pressure and RV hypertrophy. A, Mean PA pressures in rats 28 days after right lung resection and monocrotaline injection were more than 3-fold higher than in normal control rats. However, mean PA pressures 14 and 28 days after transplantation of the left lungs from pulmonary hypertensive rats into normotensive recipients were only mildly elevated from control values, confirming effective pressure unloading. Bars represent mean \pm SEM for controls $(n=4)$, lung resection and monocrotaline injection $(n=7)$, lung transplantation at 14 days $(n=$ 3 ), and lung transplantation at 28 days $(n=7)$. $\mathbf{B}, \mathrm{RV}$ hypertrophy detected as a more than 2 -fold increase in the $\mathrm{RV} / \mathrm{LV}+\mathrm{S}$ mass ratio was evident 28 days after right lung resection and monocrotaline injection relative to normal controls. However, RV hypertrophy did not occur 14 or 28 days after transplantation of hypertensive left lungs into normal rats and is consistent with the absence of pulmonary hypertension in these animals. Bars represent mean \pm SEM for controls $(n=4)$, lung resection and monocrotaline injection $(n=10)$, lung transplantation at 14 days $(n=$ 5), and lung transplantation at 28 days $(\mathrm{n}=7)$.

Right lung resection and monocrotaline injection. After the administration of anesthesia, endotracheal intubation, and ventilation, a right thoracotomy was performed in the fifth intercostal space. The right middle and lower lobe vein, artery, and bronchus were ligated with a silk suture, and the middle and lower lobes of the lung were removed. A chest tube was left in the pleural space and the thoracotomy wound was closed in layers with absorbable sutures. The alkaloid toxin monocrotaline (Sigma Chemical Co, St Louis, Mo) was dissolved in $\mathrm{HCl}(1 \mathrm{~mol} / \mathrm{L})$, diluted with sterile water (40 $\mathrm{mg} / \mathrm{mL})$, adjusted to $\mathrm{pH} 7.0$, and filtered $(0.2 \mu \mathrm{m}$ pore size $)$. Monocrotaline $(60 \mathrm{mg} / \mathrm{kg})$ was injected subcutaneously once the rats were extubated.

Left lung harvest and transplantation. Left lungs were harvested from pulmonary hypertensive rats 28 days after right lung resection and monocrotaline injection for transplantation into normotensive recipients. After anesthesia, the rats were intubated through a tracheostomy and ventilated as described above with an inspired oxygen fraction of 1. A median laparosternotomy was performed, and 300 units of heparin (Hepalean; Organon Teknika, Toronto, Canada) was injected into the inferior vena cava. For the retrieval of the heart-lung block, the inferior vena cava and the left atrial appendage were incised and a 14-gauge intravenous catheter was placed into the main PA through a right ventriculotomy. The lungs were then flushed through this catheter with $20 \mathrm{~mL}$ of a low-potassium dextran-5\% glucose preservation solution (Perferdex; Biophausia, Uppsala, Sweden) containing $500 \mu \mathrm{g} / \mathrm{L}$ of alprostadil (formerly, prostaglandin $\mathrm{E}_{1}$; ProstinVR; Upjohn, Don Mills, Ontario, Canada) from a height of $30 \mathrm{~cm}$. This solution is used in the clinical lung transplant program at the University of Toronto. Immediately after the lungs were flushed, the tracheostomy tube was clamped after inspiration to preserve the lungs in the inflated state. The heartlung block was then removed and kept on ice at $4{ }^{\circ} \mathrm{C}$ until transplantation. In preparation for transplantation, 14gauge Teflon cuffs were placed around the left PA, left pulmonary vein (PV), and the left main bronchus according to techniques previously described. ${ }^{15,16}$

Recipient animals were anesthetized, endotracheally intubated, and ventilated. A left thoracotomy was performed through the fifth intercostal space. The left lung was mobilized by transecting the pulmonary ligament. The left PA, PV, and bronchus were identified and clamped with microsurgical aneurysm clamps. The cuffs on the donor lung PA, PV, and bronchus were inserted through incisions in the corresponding recipient structures, and the anastomoses were fixed with 7-0 polypropylene ties. The warm ischemic time was standardized at 20 minutes; this is the period of time between removal of the lung from the ice bath and restoration of blood flow. Heparin (100 units) was 


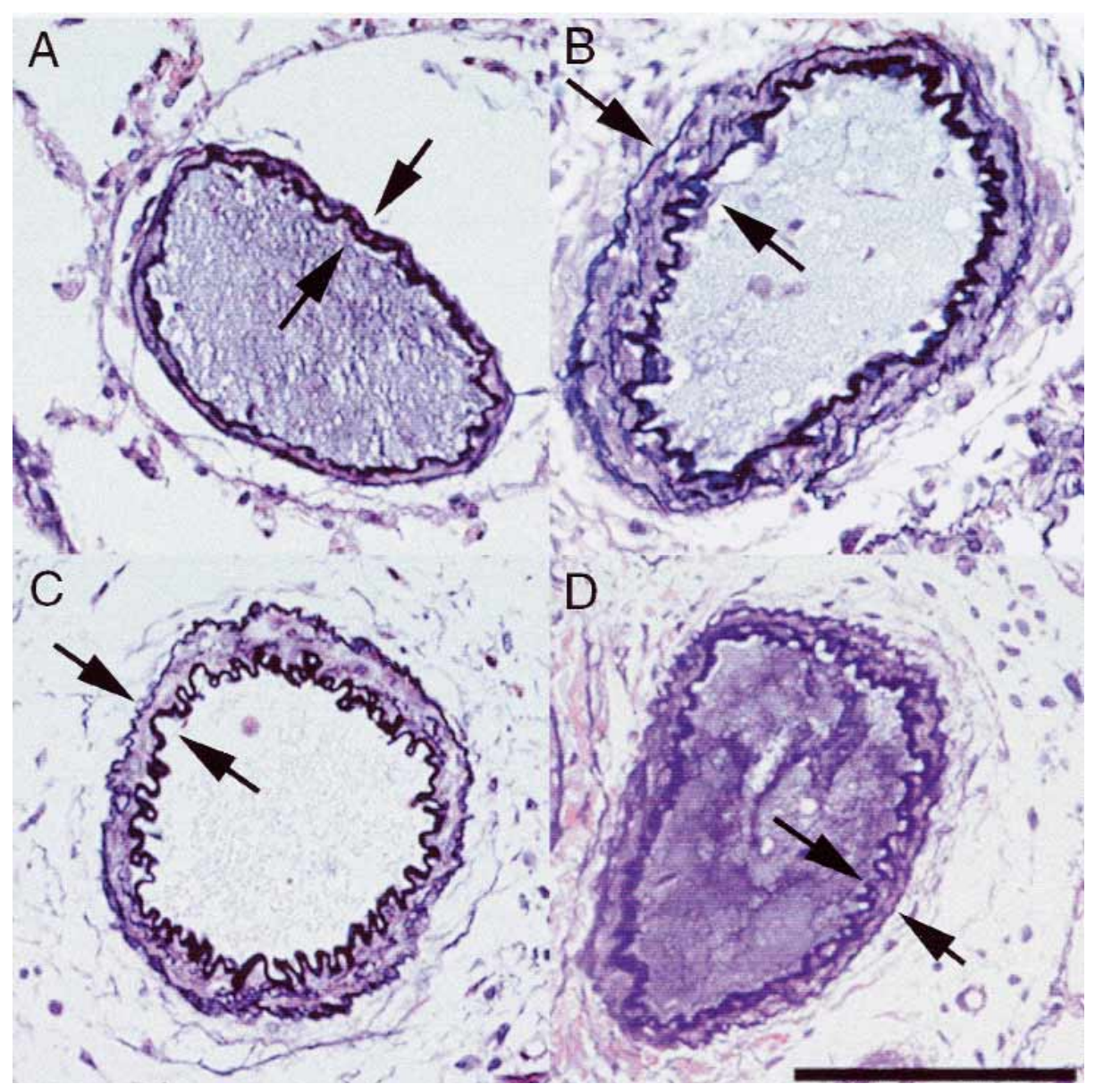

Fig 2. Percent wall thickness of muscular arteries. A to D, Representative photomicrographs of left lung sections stained with Movat pentachrome demonstrate thickening of the wall (arrows) of muscular PAs 28 days after right lung resection and monocrotaline injection $(\mathbf{B})$ relative to normal controls $(\mathbf{A})$. There was a progressive reduction in wall thickness 14 (C) and 28 (D) days after transplantation of the hypertensive lungs into normotensive recipients. Scale bar $=100 \mu \mathrm{m}$.

injected into the intrathoracic inferior vena cava, the transplanted lung was inflated by removing the clamp on the bronchus, and the lung was reperfused by unclamping the PV followed by the PA. A chest tube was left in the pleural space and the thoracotomy incision was closed in layers with absorbable sutures. Inflation of the transplanted lung was confirmed by a postoperative chest radiograph. Immunosuppressive agents were not used.

PA pressure measurement. PA pressures were measured in normal control rats, in rats 28 days after right lung resection and monocrotaline injection, and 14 and 28 days after transplantation of the hypertensive left lungs into normotensive recipient rats. Anesthesia was induced in an induction chamber with isoflurane (Zeneca, Mississauga, Ontario, Canada) and maintained with the animals breathing spontaneously through a face mask. The PA was cannulated with a silicone rubber tube $(0.64-\mathrm{mm}$ outer diameter) through the right external jugular vein by means of a previously described closed chest technique. ${ }^{17,18}$ Pressures were recorded by a transducer (MS20; Electromedics, Englewood, Colo), amplifier (Interface 4600; Gould, Inc, Cleveland, Ohio), and monitor (V1000, Gould).

Tissue preparation and assessment of RV hypertrophy. After the hemodynamic measurements were completed, a midline sternotomy was performed to expose the heart and lungs. Heparin (500 units) was injected into the RV cavity. The main PA was cannulated with polyethylene tubing (2.08$\mathrm{mm}$ outer diameter) through a right ventriculotomy, the lungs were perfused with phosphate-buffered saline solution $(\mathrm{NaCl}$ $\left.8 \mathrm{~g}, \mathrm{KCl} 0.2 \mathrm{~g}, \mathrm{Na}_{2} \mathrm{HPO}_{4} 1.4 \mathrm{~g}, \mathrm{KH}_{2} \mathrm{PO}_{4} 0.96 \mathrm{~g} / \mathrm{L}\right)$ at $20 \mathrm{~cm}$ $\mathrm{H}_{2} \mathrm{O}$ to clear the blood, and the heart and lungs were removed en bloc. The PA was perfused with a warm $\left(60^{\circ} \mathrm{C}\right)$ bariumgelatin mixture at $100 \mathrm{~cm} \mathrm{H}_{2} \mathrm{O}$ for 5 minutes to evenly distend all precapillary vessels and allow reproducible structural assessment. The lungs were then distended and fixed by perfusion with $10 \%$ formalin through the trachea for 48 

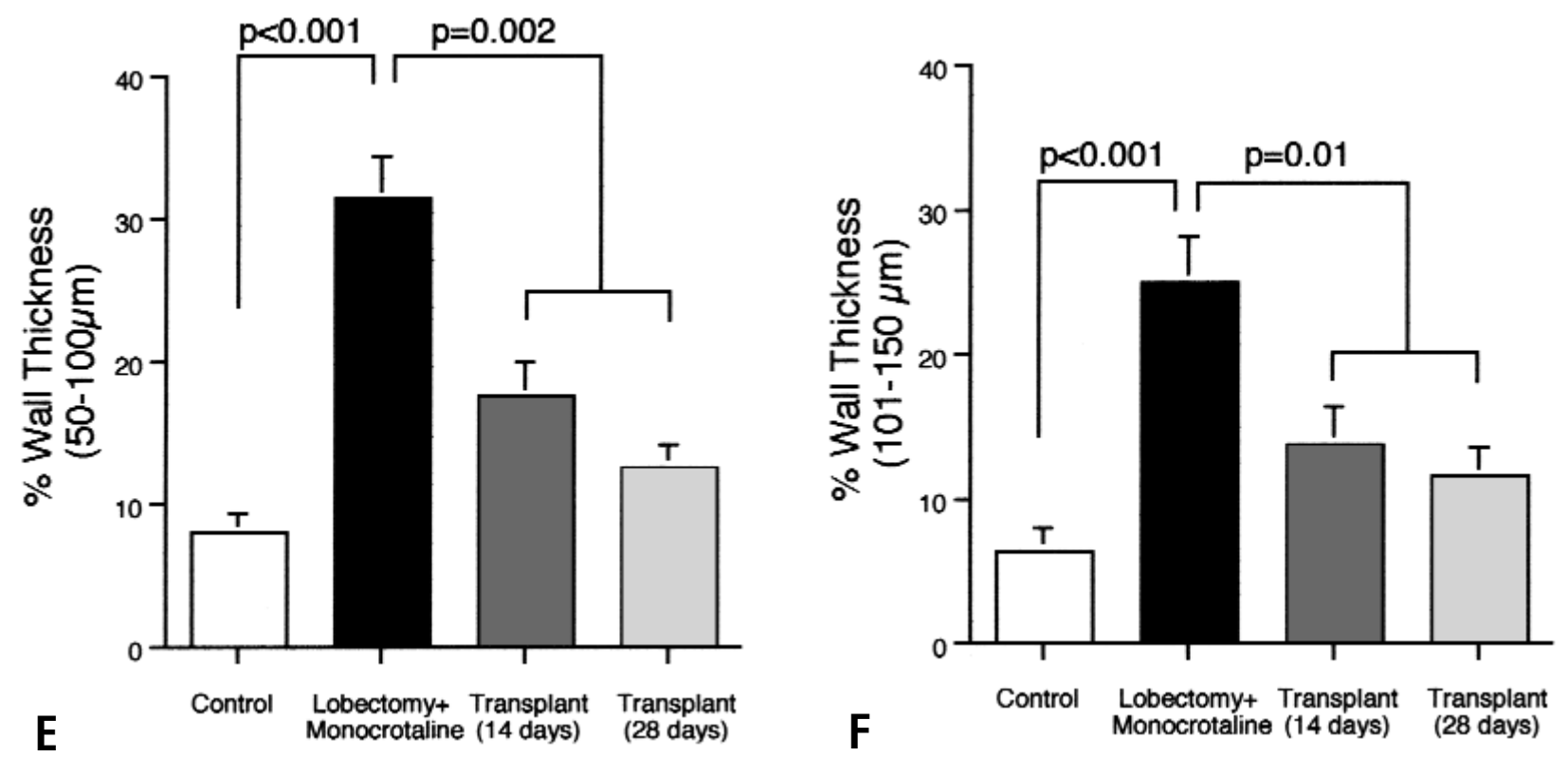

Fig 2. E and F, PA wall thickness, expressed as a percentage of external diameter as described in the "Methods" section, was increased 4-fold in 50-100- $\mu \mathrm{m}$ vessels (E) and 101-150- $\mu \mathrm{m}$ vessels (F) 28 days after right lung resection and monocrotaline injection relative to normal controls. There was progressive normalization of wall thickness 14 and 28 days after transplantation of the hypertensive left lungs into normotensive recipient rats. Bars represent mean \pm SEM for control $(n=4)$, lung resection and monocrotaline injection $(n=10)$, lung transplantation at 14 days $(n=5)$, and lung transplantation at 28 days $(n=7)$.

hours, paraffin embedded, and sectioned for light microscopy. The right ventricle (RV) was dissected from the left ventricle and septum $(\mathrm{LV}+\mathrm{S})$, both were weighed separately, and the $\mathrm{RV} / \mathrm{LV}+\mathrm{S}$ weight ratio was calculated.

Morphometric analysis of PA structure. Sections stained with Movat pentachrome were analyzed by observers blinded to the treatment group. All barium-filled arteries were assessed at $400 \times$ magnification. The external diameter and wall thickness were measured for muscular arteries of size ranges 50-100 $\mu \mathrm{m}$ and 101-150 $\mu \mathrm{m}$. Percent wall thickness $(\% \mathrm{WT})$, an index of medial hypertrophy, was calculated according to the following formula: $\% \mathrm{WT}=(2 \times \mathrm{WT}) / \mathrm{exter}-$ nal diameter $\times 100$. Extension of muscle into the normally nonmuscular PAs between 15 and $50 \mu \mathrm{m}$ in diameter was evaluated by the percentage of arteries with a complete or partial medial muscle layer. The arterial density was determined from each section by counting all arteries and alveoli in each of 10 random fields at $200 \times$ magnification. Arterial density was expressed as the number of arteries per 100 alveoli.

Evaluation of cellular proliferation. Cellular proliferation was evaluated by immunohistochemical staining with a monoclonal antibody against the proliferating cell nuclear antigen (PCNA). To detect PCNA, we incubated deparaffinized tissue sections for 60 minutes at room temperature with nuclease (Cell proliferation kit; Amersham International, Buckinghamshire, United Kingdom). Sections were incubated overnight at $4^{\circ} \mathrm{C}$ with a monoclonal antibody against PCNA (PC10, dilution 1:100 in phosphate-buffered saline solution; DAKO Corporation, Carpinteria, Calif), then with a peroxidase-conjugated secondary antibody (15 $\mu \mathrm{L} / \mathrm{mL}$; Amersham International), developed with 3,3'diaminobenzidine (Sigma Chemical Company, St Louis, Mo) and counterstained with eosin. Additional sections were incubated with nonimmune mouse immunoglobulin $\mathrm{G}$ as a negative control and showed no background staining.

PCNA-positive cells were quantified in a blinded fashion by counting the number of positively stained nuclei in 10 randomly selected arteries between 15 and $100 \mu \mathrm{m}$ in diameter. The total number of nuclei was determined in the same arteries from the corresponding hematoxylin and eosinstained serial sections, and the percentage of PCNA-positive cells relative to total cell number was calculated.

Data analysis. Data are expressed as mean \pm SEM. Statistical significance was assessed by means of a 1-way analysis of variance with post hoc testing by the Fisher protected least significant difference method except for bodyweight change, which was assessed by means of a paired $t$ test.

\section{Results}

Perioperative survival. Normal rats $(n=4)$ served as one control group and did not undergo any treatment. Of the 37 rats undergoing pulmonary resection and monocrotaline injection, 7 did not survive the procedure. By postoperative day 28, the 30 surviving rats exhibited signs of respiratory distress and were failing 


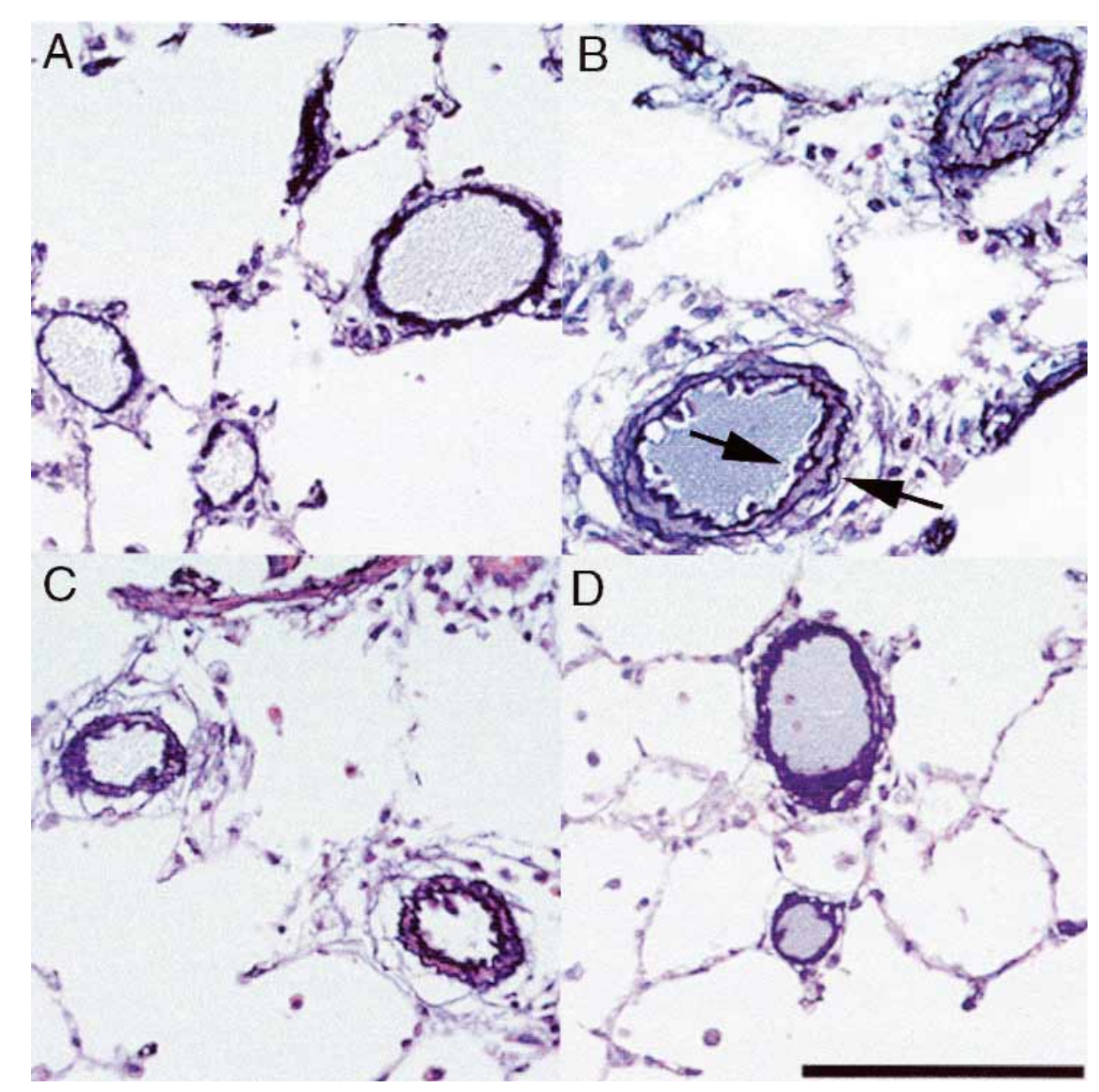

Fig 3. Muscularization of peripheral PAs and arterial density. A to D, Representative photomicrographs of left lung sections stained with Movat pentachrome demonstrate extension of smooth muscle (arrows) into small peripheral PAs 28 days after right lung resection and monocrotaline injection (B) relative to the normally nonmuscular arteries seen in normal control rats (A). There was a progressive decrease in arterial muscularization 14 (C) and 28 (D) days after transplantation of hypertensive left lungs into normotensive recipients. Scale bar $=100 \mu \mathrm{m}$.

to thrive as indicated by a $5 \%$ reduction from their preoperative weight $(345 \pm 9$ vs $363 \pm 8 \mathrm{~g}, P=.005, \mathrm{n}=$ $30)$. One third $(\mathrm{n}=10)$ were randomly selected as a baseline hypertensive group and the remaining rats $(\mathrm{n}=$ 20) underwent transplantation of the hypertensive left lung into normotensive recipients. The ischemic period between the time the lung was harvested and blood flow was restored was $98 \pm 5$ minutes. Six recipient rats did not survive the transplant procedure. The surviving recipient rats were killed at $14(\mathrm{n}=5)$ and 28 days $(\mathrm{n}=$ 7) after transplantation.

PA pressure and RV hypertrophy. Mean PA pressures were elevated more than 3 -fold in rats 28 days after right lung resection and monocrotaline injection relative to normal controls (Fig 1, A). Pulmonary hypertension was associated with marked RV hypertrophy, indicated by a more than 2 -fold increase in the $\mathrm{RV} / \mathrm{LV}+\mathrm{S}$ weight ratio (Fig 2, B). The hypertensive left lungs explanted from rats 28 days after right lung resection and monocrotaline injection were transplanted into normotensive recipients. We then confirmed hemodynamic unloading of the transplanted lung by the observation that mean PA pressures were not significantly different from control values 14 days after transplantation and were only mildly elevated by day 28 (Fig $1, A, P=.02$ for transplant [28 days] vs control). Hemodynamic unloading was further supported by the observation that RV hypertrophy did not develop in the recipient rats 14 or 28 days after transplantation (Fig 1,B).

Pulmonary vascular remodeling. We next evaluated PA structure in the hypertensive lungs from rats 28 days after right lung resection and monocrotaline injection. There was a 4 -fold increase in percent wall thickness, an index of medial hypertrophy, in the hyperten- 

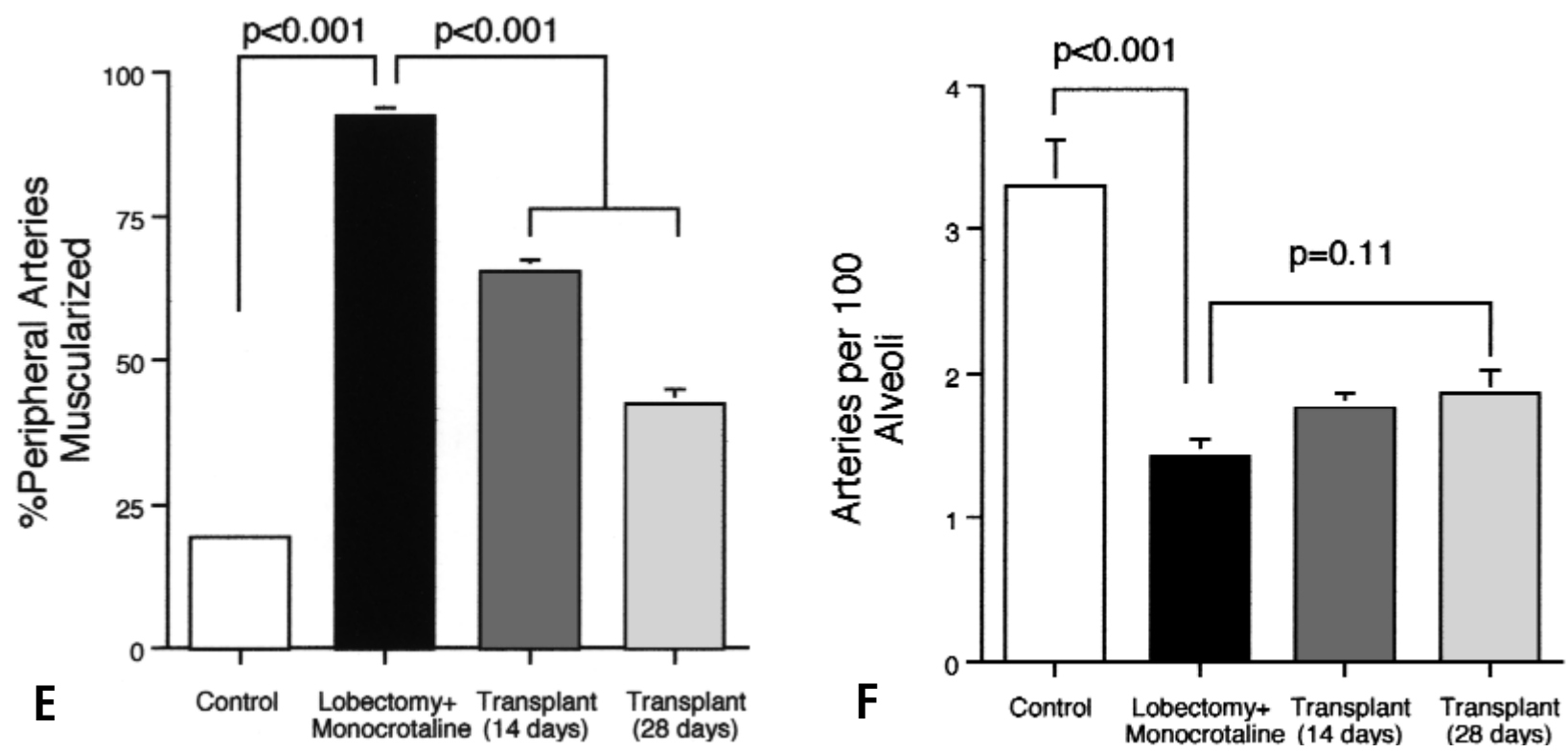

Fig 3. E, There was a more than 4-fold increase in the percentage of partially or completely muscularized arteries $15-50 \mu \mathrm{m}$ in diameter 28 days after right lung resection and monocrotaline injection relative to normal controls. A progressive reduction in arterial muscularization was evident 14 and 28 days after transplantation of the hypertensive left lungs into normotensive recipients. F, Pulmonary arterial density (arteries/100 alveoli) was reduced by $58 \% 28$ days after right lung resection and monocrotaline injection. Arterial density remained low 14 and 28 days after transplantation of the hypertensive left lungs into normotensive recipients. Bars represent mean \pm SEM for control $(n=4)$, lung resection and monocrotaline injection $(n=10)$, transplantation at 14 days $(n=5)$, and transplantation at 28 days $(n=7)$.

sive lungs relative to normal controls for both the 50$100-\mu \mathrm{m}$ and 101-150- $\mu \mathrm{m}$ vessel size ranges (Fig 2, A and $B$, and Fig 2, $E$ and $F$ ). However, there was progressive normalization of wall thickness 14 and 28 days after unloading of the hypertensive left lungs by transplantation into normotensive recipients (Fig 2, $C$ and $D$, and Fig 2, $E$ and $F$ ).

Small peripheral PAs (15-50 $\mu \mathrm{m}$ in diameter) in normal lungs are nonmuscular in that they lack a smooth muscle layer, as shown in Fig 3, A. However, with the development of obstructive pulmonary vascular disease, the peripheral PAs become muscularized, as seen in the lungs from rats 28 days after right lung resection and monocrotaline injection (Fig 3, B). We quantified the number of small PAs that were partially or completely muscularized and found a more than 4-fold increase in the hypertensive lungs relative to normal controls (Fig 3, E). However, when the hypertensive lungs were unloaded by transplantation into normotensive recipients, regression of the abnormal PA muscularization was apparent 14 and 28 days later (Fig 3,C and $D$, and Fig 3,E).

Because obstructive pulmonary vascular disease is also characterized by loss of peripheral PAs, we next evaluated arterial density by quantifying the number of arteries per 100 alveoli in the lung sections. There was a 58\% reduction in arterial density in lungs from rats 28 days after right lung resection and monocrotaline injection relative to normal controls (Fig 3,F). Whereas hemodynamic unloading induced marked reductions in medial hypertrophy and peripheral muscularization, arterial density was only modestly improved either 14 or 28 days after transplantation (Fig 3, F).

We next examined the right lung of recipient rats to evaluate the impact of transplanting the hypertensive left lung on the structure of the remaining pulmonary vasculature. Arterial density remained normal $(3.5 \pm$ 0.4 and $3.3 \pm 0.2$ arteries per 100 alveoli at 14 and 28 days) relative to untreated controls $(3.3 \pm 0.3)$. Medial thickness was also normal $(5.5 \% \pm 0.2 \%$ and $6.8 \% \pm$ $0.6 \%$ wall thickness at 14 and 28 days) relative to untreated controls $(7.2 \% \pm 1.5 \%)$, as was muscularization of peripheral arteries (17.5\% $\pm 2.3 \%$ [14 days] and $20.0 \% \pm 2.9 \%$ [ 28 days] vs $19.4 \pm 1.2 \%$ [control]).

Cellular proliferation. One mechanism contributing to medial hypertrophy and peripheral muscularization in obstructive pulmonary vascular disease is proliferation of smooth muscle cells in the PA wall. We there- 


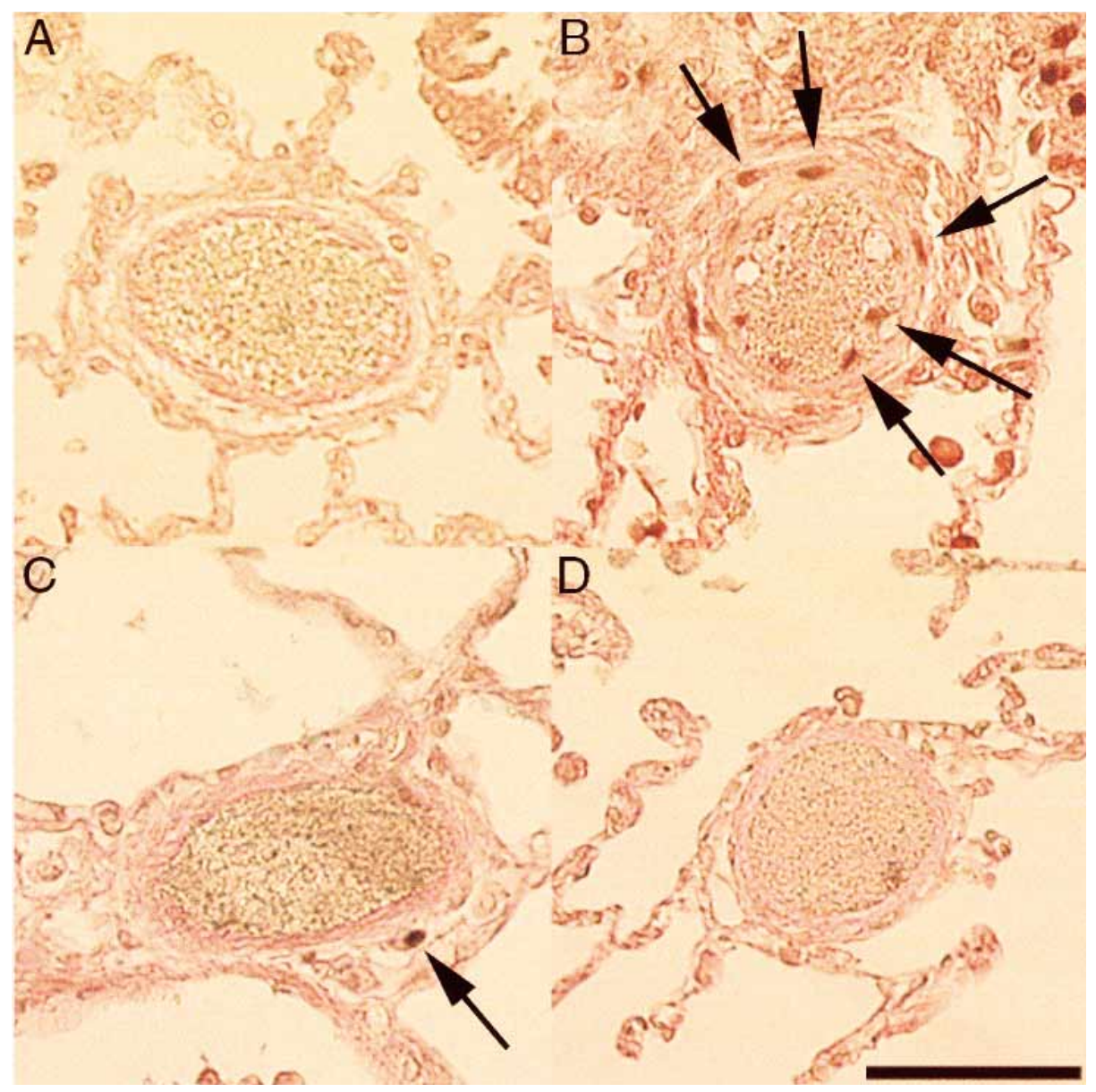

Fig 4. Cellular proliferation. A to D, Representative photomicrographs of immunoperoxidase staining of left lung sections with a monoclonal antibody against PCNA demonstrate positive nuclei (arrows) suggesting increased cellular proliferation in the PA wall 28 days after right lung resection and monocrotaline injection (B) relative to normal controls (A). Positive nuclei were markedly reduced 14 (C) and 28 (D) days after transplantation of the hypertensive left lungs into normotensive recipients. Scale bar $=50 \mu \mathrm{m}$.

fore looked for evidence of PA cellular proliferation by immunoreactivity for the PCNA. Indices of PCNA-positive cells are low in normal PAs (Fig 4, A, and Fig 4E) in contrast to a more than 9 -fold increase 28 days after right lung resection and monocrotaline injection (Fig 4, $B$, and Fig 4E). When the hypertensive lungs were unloaded by transplantation into normotensive recipients, the number of PCNA-positive cells returned toward baseline levels by days 14 and 28 (Fig 4, $C$ and $D$, and Fig 4E). We also examined the lungs for evidence of cellular apoptosis using the in situ TUNEL (terminal deoxynucleotidyl transferase-mediated dUTP nick end labeling) assay; however, none was detected in PAs either 14 or 28 days after transplantation (data not shown).

\section{Discussion}

Patients with congenital heart defects and with a fixed increase in pulmonary vascular resistance as a result of obstructive pulmonary vascular disease represent a therapeutic challenge. These patients may not be candidates for surgical correction of their congenital heart lesion because of the risk of postoperative pulmonary hypertension and RV failure. However, if the structural changes in the pulmonary circulation associated with obstructive pulmonary vascular disease could be reversed, surgical correction of the heart defect might be accomplished with acceptable risk.

Our laboratory has studied the pathobiology of the development and regression of pulmonary vascular disease. We have found that PAs from rats with experi- 


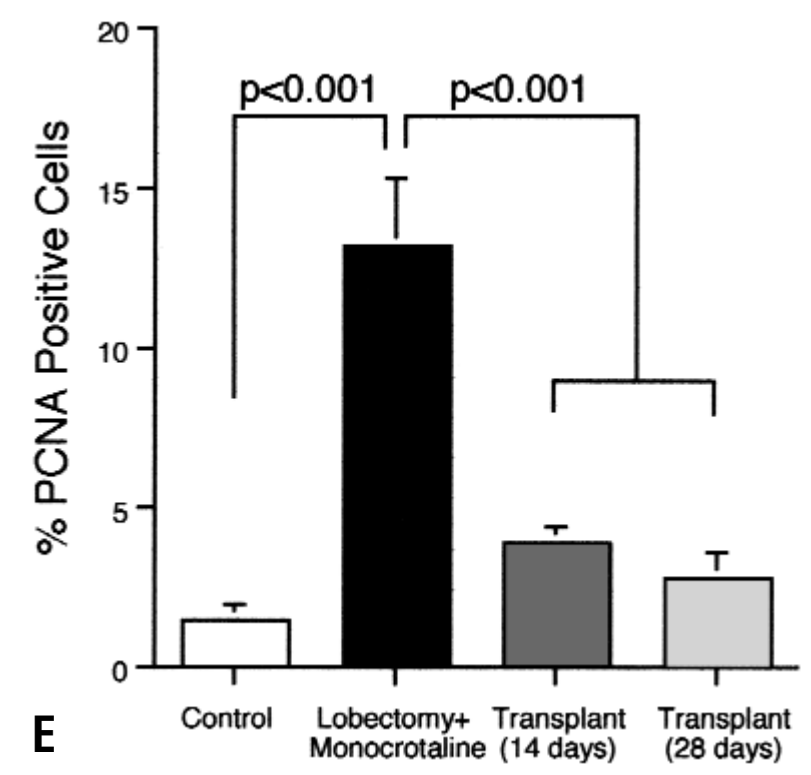

Fig 4E. The percentage of nuclei stained positive with an anti-PCNA antibody was elevated more than 9-fold in the PA wall of rats 28 days after right lung resection and monocrotaline injection relative to normal controls. The percentage of PCNA-positive cells in the PAs of hypertensive lungs returned to control values 14 and 28 days after transplantation into normotensive recipients. Bars represent mean \pm SEM from control $(\mathrm{n}=4)$, lung resection and monocrotaline injection $(n=10)$, lung transplantation at 14 days $(n=5)$, and lung transplantation at 28 days $(n=7)$.

mental pulmonary vascular disease hypertrophy as a result of smooth muscle cell proliferation when grown under tension in an organ culture model ${ }^{10}$ Furthermore, when the tension is "unloaded," smooth muscle cell proliferation stops and apoptosis is induced, leading to regression of PA hypertrophy. We therefore hypothesized that a similar mechanism may be active in vivo and could potentially be exploited to reverse pulmonary vascular disease in patients with congenital heart defects. To test this hypothesis, we developed a model in which hypertensive rat lungs with experimental pulmonary vascular disease were hemodynamically unloaded by transplantation into syngeneic, normotensive recipient rats.

In these experiments we show that advanced obstructive pulmonary vascular disease develops in rats 28 days after right lung resection and monocrotaline injection. Furthermore, unloading the pulmonary circulation of the hypertensive lungs by transplantation into normotensive recipient rats results in improvement in medial hypertrophy and peripheral muscularization associated with a reduction in PA cellular proliferation rates. However PA density, although somewhat improved, remains markedly below baseline levels.

In this model, unloading of the hypertensive lungs is accomplished by transplantation into normotensive recipients. To maximize the potential for unloading, we transplanted hypertensive lungs into healthy recipients rather than the usual clinical situation of transplanting a normal lung into a hypertensive recipient. We have documented that PA pressures are normal 14 days after transplantation and only mildly elevated by day 28 . This observed increase in PA pressure after transplantation is not due to the development of vascular disease in the native pulmonary circulation, because arterial density, muscularization, and medial thickness all remained normal. However, it may be due to an improvement in cardiac output as the animals recover from the early postoperative period or to the development of some degree of stenosis at the PA anastomoses, even though all were patent at the time of tissue harvest and barium injection.

Consistent with an absence of major pulmonary hypertension, RV hypertrophy did not develop in the recipient animals. We presume that pulmonary blood flow to the transplanted lung is also reduced as a result of shunting toward the normal lung, although this was not measured directly. We have shown improvement in the structure of diseased PAs after unloading. However, we have not measured pulmonary vascular resistance, which must be reduced to have a favorable impact on the risk of corrective surgery in patients with congenital heart defects.

Arterial density was reduced with the development of obstructive pulmonary vascular disease in this model. 
We observed a modest improvement in arterial density 28 days after unloading of the hypertensive lungs; however, it was still markedly depressed relative to that of normal controls. Reduced arterial density contributes to the fixed increase in pulmonary vascular resistance in patients with obstructive pulmonary vascular disease. ${ }^{6-9}$ Although arterial density could continue to improve beyond 28 days, the results with unloading of the pulmonary circulation may be limited by a persistent decrease in arterial density. Therefore, concomitant angiogenic therapy ${ }^{19}$ could theoretically be useful in addition to hemodynamic unloading as a method of restoring the PA structure.

Regression of PA medial hypertrophy in organ culture is associated with the induction of apoptosis, which was detected up to 1 week after stress unloading. ${ }^{10,20}$ However, we did not observe high levels of apoptosis in PAs after unloading in this in vivo model (data not shown). Apoptosis may therefore be an early event that occurs before day 14 , which is the earliest time point that we have examined. Further reductions in medial hypertrophy and peripheral muscularization may be due to low levels of ongoing apoptosis or resorption of extracellular matrix components. ${ }^{20}$

PA banding has been proposed as one method of unloading the pulmonary circulation to induce regression of pulmonary vascular disease. ${ }^{11}$ However, unloading of the pulmonary circulation by banding of the main PA is limited by the degree of cyanosis that results from increased right-to-left shunting across cardiac septal defects. Therefore, alternative strategies will likely be required to make this approach clinically applicable. For example, unilateral PA banding might allow for regression of pulmonary vascular disease in the banded lung while maintaining adequate flow to the contralateral lung. Improved PA structure in the banded lung alone may be sufficient to permit subsequent surgical repair of the heart defect with a reasonable safety margin while avoiding the consequences of banding the main PA. Furthermore, extracorporeal membrane oxygenation has been used to successfully reduce perioperative PA pressures ${ }^{21}$ and could in theory promote recovery of pulmonary vascular structure.

Patients with pulmonary vascular disease as a result of a cardiac defect can be treated by single lung transplantation with cardiac repair. This study supports the intriguing concept that a single lung transplant with cardiac repair will not only provide immediate life-saving therapy but could also unload the remaining native lung, possibly leading to reversal of the pulmonary vascular changes. This clinical scenario may be the only situation in which the concept of hemodynamic unloading to reverse what is conventionally thought of as irreversible pulmonary vascular disease can be safely studied in human beings.

Because of our understanding of the cellular mechanisms involved, we have been able to develop therapeutic strategies that target the critical biologic processes directly, in addition to reversing pulmonary vascular disease by hemodynamic unloading. For example, we have identified that cellular proliferation in experimental pulmonary vascular disease is mediated by the activity of proteolytic enzymes including serine elastases and matrix metallo-proteinases. These enzymes release growth factors and up-regulate $\alpha v \beta 3$ ligands such as tenascin $\mathrm{C}$, which in turn promote the proliferative response by activating growth factor receptors. ${ }^{23}$ The results of targeting these enzymes with specific inhibitors are that smooth muscle cell proliferation is arrested, apoptosis is induced, extracellular matrix is resorbed, and the pulmonary vascular changes regress in both organ culture ${ }^{21}$ and whole animal models. ${ }^{14}$ Therefore, other strategies may be useful in conjunction with or as an alternative to hemodynamic unloading to reverse pulmonary vascular disease.

Applicability of these observations to human pulmonary vascular disease is limited by the suitability of the model. Lung resection to increase blood flow in the remaining PA modifies the response to monocrotaline so that gene expression and remodeling closely resemble the human disease. ${ }^{12,13}$ However, significant differences in the time course and pathologic findings still exist. A second limitation relates to the use of transplantation as a method to unload the pulmonary circulation in which we could not control for the potential impact of denervation and disruption of bronchial blood supply.

In summary, strategies to unload the pulmonary circulation or to activate the critical mechanisms by means of a biologic approach may improve the structural changes associated with obstructive pulmonary vascular disease in patients with congenital heart defects. Angiogenic therapies may afford additional benefit by improving arterial density, which appears less responsive to unloading than does medial hypertrophy or muscularization of peripheral PAs. Therefore, a multifaceted approach to this challenging problem seems promising and should be critically evaluated in an attempt to improve the outcome for these patients.

We thank Dr Michael Borger, who reviewed the statistical analysis, and J. Jowlabar, J. Matthews, and J. Edwards for secretarial assistance. 


\section{REFERENCES}

1. Fremes SE, Patterson GA, Williams WG, Goldman BS, Todd TR, Maurer J. Single lung transplantation and closure of patent ductus arteriosus for Eisenmenger's syndrome. J Thorac Cardiovasc Surg 1990;100:1-5.

2. Spray TL, Mallory GB, Canter CE, Huddleston CB, Kaiser LR. Pediatric lung transplantation for pulmonary hypertension and congenital heart disease. Ann Thorac Surg 1992;54:216-23.

3. McGregor CG, Jamieson SW, Baldwin JC, Burke CM, Dawkins $\mathrm{KD}$, Stinson EB, et al. Combined heart-lung transplantation for end-stage Eisenmenger's syndrome. J Thorac Cardiovasc Surg 1986;91:443-50.

4. Aeba R, Griffith BP, Hardesty RL, Kormos RL, Armitage JM. Isolated lung transplantation for patients with Eisenmenger's syndrome. Circulation 1993;88(Suppl):II-452-5.

5. Rosenzweig EB, Kerstein D, Barst RJ. Long-term prostacyclin for pulmonary hypertension with associated congenital heart defects. Circulation 1999;99:1858-65.

6. Meyrick B, Reid L. Ultrastructural findings in lung biopsy material from children with congenital heart defects. Am J Pathol 1980;101:527-42.

7. Meyrick B, Reid L. Pulmonary hypertension: anatomic and physiologic correlates. Clin Chest Med 1983;4:199-217.

8. Rabinovitch M, Haworth SG, Castaneda AR, Nadas AS, Reid LM. Lung biopsy in congenital heart disease: a morphometric approach to pulmonary vascular disease. Circulation 1978; 58:1107-22.

9. Rabinovitch M, Keane JF, Norwood WI, Castaneda AR, Reid L. Vascular structure in lung tissue obtained at biopsy correlated with pulmonary hemodynamic findings after repair of congenital heart defects. Circulation 1984;69:655-67.

10. Cowan KN, Jones PL, Rabinovitch M. Regression of hypertrophied rat pulmonary arteries in organ culture is associated with suppression of proteolytic activity, inhibition of tenascin-C, and smooth muscle cell apoptosis. Circ Res 1999;84:1223-33.

11. Wagenvoort CA, Wagenvoort N, Draulans-Noe Y. Reversibility of plexogenic pulmonary arteriopathy following banding of the pulmonary artery. J Thorac Cardiovasc Surg 1984;87:876-86.

12. Tanaka Y, Schuster DP, Davis EC, Patterson GA, Botney MD. The role of vascular injury and hemodynamics in rat pulmonary artery remodeling. J Clin Invest 1996;98:434-42.

13. Okada K, Tanaka Y, Bernstein M, Zhang W, Patterson GA, Botney MD. Pulmonary hemodynamics modify the rat pulmonary artery response to injury: a neointimal model of pulmonary hypertension. Am J Pathol 1997;151:1019-25.

14. Cowan KN, Heilbut A, Humpl T, Rabinovitch M. Complete reversal of fatal pulmonary hypertension in rats with a serine elastase inhibitor. Nat Med 2000;6:698-702.

15. Mizuta T, Kawaguchi A, Nakahara K, Kawashima Y. Simplified rat lung transplantation using a cuff technique. J Thorac Cardiovasc Surg 1989;97:578-81.

16. Reis A, Giaid A, Serrick C, Shennib H. Improved outcome of rat lung transplantation with modification of the nonsuture external cuff technique. J Heart Lung Transplant 1995;14:274-9.
17. Herget J, Palecek F. Pulmonary arterial blood pressure in closed chest rats: changes after catecholamines, histamine and serotonin. Arch Int Pharmacodyn Ther 1972;198:107-17.

18. Rabinovitch M, Gamble W, Nadas AS, Miettinen OS, Reid L. Rat pulmonary circulation after chronic hypoxia: hemodynamic and structural features. Am J Physiol 1979;236:H818-27.

19. Campbell AIM, Zhao YD, Robb MJJ, Stewart DJ. Cell-based gene transfer of angiopoietin-1 reduces monocrotaline-induced pulmonary hypertension. Circulation 1999;100(Suppl):I-476.

20. Cowan KN, Jones PL, Rabinovitch M. Elastase and matrix metalloproteinase inhibitors induce regression, and tenascin- $\mathrm{C}$ antisense prevents progression, of vascular disease. J Clin Invest 2000;105:21-34.

21. Dhillon R, Pearson G, Firmin R, Chan K, Leanage R. Extracorporeal membrane oxygenation and the treatment of critical pulmonary hypertension in congenital heart disease. Eur $\mathbf{J}$ Cardiothorac Surg 1995;9:553-6.

22. Thompson KE, Rabinovitch M. Human leukocyte elastase mediates release of extracellular matrix-bound bFGF in vascular smooth muscle cell cultures. Mol Biol Cell 1994;5(suppl):378a.

23. Jones PL, Rabinovitch M. Tenascin-C induced with progressive pulmonary vascular disease in rats is functionally related to increased smooth muscle cell proliferation. Circ Res 1996;79:1131-42.

24. Jones PL, Crack J, Rabinovitch M. Regulation of tenascin-C, a vascular smooth muscle cell survival factor that interacts with the $\alpha v \beta 3$ integrin to promote epidermal growth factor receptor phosphorylation and growth. J Cell Biol 1997;139:279-93.

\section{Discussion}

Dr Fred A. Crawford (Charleston, SC). Dr O'Blenes, pulmonary hypertension has been documented to occur and progress after the successful repair of anomalies such as ventricular septal defects and complete atrioventricular septal defects. Presumably these lungs have been unloaded by such a repair. How do you explain that in light of your findings?

Dr O'Blenes. In the experiment we presented, the PA pressures were unloaded to normal pressure ranges. If you were to repair a ventricular septal defect in a patient with fixed pulmonary hypertension, for example, you would expect PA pressures to remain high in the postoperative period. That could explain why pulmonary hypertension might progress in that group of patients: the shunt is being closed but the pressure to that vasculature is not being restricted.

Another possibility is that some patients may be predisposed to the development of severe pulmonary hypertension, not purely on the basis of increased flow. They may have a predisposition to a sort of primary pulmonary hypertension that will progress despite the repair. 